\title{
Efficient State-Diagram Construction Methods for Software Pipelining
}

\author{
Chihong Zhang ${ }^{1}$, Ramaswamy Govindarajan ${ }^{2}$, Sean Ryan ${ }^{1}$, and \\ Guang R. Gao ${ }^{1}$ \\ 1 Dept. of Electric and Computer Engineering, Univ. of Delaware \\ Newark, DE 19711, USA \\ \{czhang, ryan, ggao\}@capsl.udel.edu \\ 2 Supercomputer Edn. \& Res. Centre, Indian Institute of Science \\ Bangalore, 560 012, India \\ govind@serc.iisc.ernet.in
}

\begin{abstract}
State diagram based approach has been proposed as an effective way to model resource constraints in traditional instruction scheduling and software pipelining methods. However, the constructed state diagram for software pipelining method (i) is very large and (ii) contains significant amount of replicated, and hence redundant, information on legal latency sequences. As a result, the construction of state diagrams can take very large computation time.

In this paper, we propose two methods for the efficient construction of state diagrams. In the first method, we relate the construction of state diagram to a well-known problem in graph theory, namely the enumeration of maximal independent sets of a graph. This facilitates the use of an existing algorithm as a direct method for constructing distinct latency sequences. The second method is a heuristic approach which exploits the structure of state diagram construction to eliminate redundancy at the earliest opportunity in an aggressive fashion. The heuristic method uses a surprisingly simple check which is formally shown to completely eliminate redundancy in the state diagram. From our experimental results on two real architectures, both of the two methods show a great reduction in state diagram construction time.
\end{abstract}

\section{Introduction}

Recent studies on modulo scheduling, an instruction scheduling method for loops $[9,10,15,16,3]$, in a production compiler has reported significant improvement (up to $35 \%$ ) in the overall runtime for a suite of SPEC floating point benchmark programs [17]. On the other hand, rapid advances in VLSI technology and computer architecture present an important challenge for compiler designers: a modulo scheduler must be able to handle machine resource constraints much more complex than before and may need to search for an increasingly larger number of schedules before a desirable one is chosen. Therefore, in exploiting the advantage of modulo scheduling in a production compiler, it is important 
to be able to handle complex resource constraints efficiently and find a good modulo schedule very quickly.

Proebsting and Fraser proposed an interesting approach that uses finite state automata for modeling complex resource constraints in instruction scheduling [14]. Their approach was based on [12] and subsequently improved and applied to production compilers in [2]. This approach was extended to software pipelining methods in $[6,7]$. This paper focuses on the efficient construction of finite state automata for software pipelining methods. In the above methods $[2,6,7,12,14]$, processor resources are modeled using a finite state automata (or state diagram) which is constructed from the resource usage table for each instruction (class). Each path in the state diagram represents a legal latency sequence which could be directly used by the scheduler for modeling resource contention. The construction of the state diagram is typically done off-line and stored in some form so that the instruction scheduler, at the schedule time, only need to read this information. This has effectively reduced the problem of checking structural hazards in the scheduling method to a fast table lookup, resulting in several fold speedup $[2,7,12]$. In particular, the enhanced Co-Scheduling method, a state diagram based modulo scheduling method, reports a 2 -fold speedup in the scheduling time (time to construct the software pipelined schedule) [7].

There are two major challenges facing this Co-scheduling method: (i)the huge size of the state diagram and (ii)the the huge amount of redundant information (paths in the state diagram, which will be used to guide the Co-Scheduling) inside the naively constructed state diagram. A practical solution to the first problem is to construct only a large enough (instead of complete) set of nonredundant state diagram information. However, the huge amount of redundancy makes the construction time extremely long.

To illustrate the above problem, we present several experimental evidences. In one experiment conducted by us, the state diagram for the DEC Alpha 21064 processor with an initiation interval (II) equal to 3, contained 224,400 latency sequences, out of which only 30 were distinct. In another experiment, the state diagram for an $\mathbf{I I}=16$ contained more than 74 Million $(74,183,493)$ distinct paths $^{1}$. Lastly, to generate 100,000 distinct latency sequences in the above state diagram, it took more than 24 hours on an UltraSparc machine. This obviously makes the Co-Scheduling approach impractical. The previous effort on reduced state diagram $[7,8]$ has met with only very limited success.

In this paper, we propose two methods to drastically reduce the construction time of state diagrams by eliminating the redundancy cleverly during the construction. The first of these methods relates the construction to a well-known problem in graph theory, namely the enumeration of maximal independent sets of an interference graph. This facilitates the use of an existing enumeration algorithm as a direct fast method for constructing all latency sequences. We refer to this method as the Enumeration of Maximal Independent Set (E-MIS) method.

\footnotetext{
${ }^{1}$ The number of actual paths (including the redundant ones) is far too many to count and generate!
} 
Two major advantages of this method are that it is a direct method and it generates only distinct (non-redundant) latency sequences.

The second method uses a heuristic approach to eliminate redundant paths by exploiting the structure of state diagram construction and by employing an aggressive redundance removal approach. This is accomplished by enforcing a surprisingly simple redundance constraint which eliminates completely all redundant paths. We refer to this method as the Redundancy Prevention (RP) method, as it identifies, at the earliest in the construction process, states which could cause redundancy and prunes them aggressively. We formally establish that the proposed heuristic results in redundance-free state diagram. However, the redundance constraint used by the heuristic is only a necessary (but not sufficient) condition. As a consequence, the aggressive pruning may eliminate some non-redundant paths as well. However, we find that the RP method to work well in practice.

We compare the efficiency of the proposed methods with that of the reduced state-diagram construction (RD) method in modeling two real processors, namely the DEC Alpha 21064 processor and the Cydra VLIW processor [3]. Our experimental results reveal that the proposed methods result in significant reduction in the construction time by about 3 to 4 orders of magnitude which means we can provide a reasonable amount of distinct paths within minutes instead of days. Another interesting observation made from our experiments is that the RP method, though a heuristic approach which can possibly eliminate nonredundant paths, does reasonably well in enumerating all non-redundant paths. In fact, for the two processors modeled and for small values of II less than 16, it did not miss a single path. Lastly, we use the latency sequences constructed by RP and E-MIS methods in the Co-Scheduling framework to construct software pipelined schedules. Initial experiments reveal that the proposed methods do seem to perform competitively, and in a reasonable computation time. This provides an empirical evidence for the use of state diagram approach as a practical and efficient method in software pipelining methods.

In Section 2, we present a brief review of the state diagram based software pipelining method. The problem formulation and the proposed approaches are informally discussed in Section 2.2. Section 3 and 4 respectively present the details of the two proposed methods. In Section 5 we compare the performance of E-MIS and RP methods with the reduced state diagram construction. Concluding remarks are provided in Section 6.

\section{Background and Motivation}

Software pipelining has been found to be an efficient compilation technique that results in significant performance improvement at runtime $[9,10,15,16]$. Modeling of resource constraints (or structural hazards) in software pipelining is becoming increasingly complex in modern processor architectures. However, an efficient resource model is crucial for the success of the scheduling method $[2,4,6,14]$. In this section, first, we review the state diagram based resource model used in 
software pipelining [6]. Subsequently we motivate our approaches to the efficient construction of state diagram.

\section{$2.1 \quad$ Background}

Conventional approaches to model resource constraints uses a simple reservation table (see Figure 1(a)) $[13,10,9,15,16]$. The state diagram approach is a systematic and effective way to represent the resource usages and conflicts when multiple operations of a loop are scheduled [6]. Such an approach is efficient as it uses the underlying notions of forbidden (permissible) latencies in the classical pipeline theory.

A state diagram represents legal latency sequences that do not cause structural hazards in the pipeline. Each state in a state diagram represents the current state of the pipeline (operating under software pipelining with an Initiation Interval (II)). Associated with each state is the set of permissible latencies at which new initiations can be made from the current state. An edge from state $S$ to $S^{\prime}$ in the state diagram represents an initiation with a latency $l$ cycles after $S$. If the current state $S$ has permissible latencies $\left\{p_{1}, p_{2}, \cdots, p_{k}\right\}$, then the new state $S^{\prime}$ will have permissible latencies $p=\left(\left(p_{i}-l\right) \bmod \mathbf{I I}\right)$, for each $i \in[1, k]$, provided $p$ is a permissible latency in the initial state $S_{0}$. The state diagram for the above reservation table is shown in Figure 2. For more detailed information on related concepts and the construction of state diagrams see $[6,7,8]$.

\begin{tabular}{|c|c|c|c|c|}
\hline Stage & \multicolumn{4}{|c|}{ Time Steps } \\
\cline { 2 - 4 } & 0 & 1 & 2 & 3 \\
\hline 1 & $\mathrm{x}$ & & \\
\hline 2 & & $\mathrm{x}$ & $\mathrm{x}$ & \\
\hline 3 & & & $\mathrm{x}$ \\
\hline
\end{tabular}

(a) Reservation Table

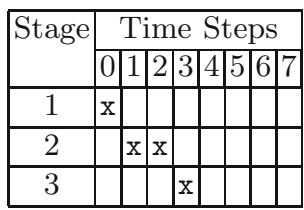

(b) Resource Usage when $\mathbf{I I}=8$.

Fig. 1. A Reservation Table and its Resource Usage for an II

A path $S_{0} \stackrel{p_{1}}{\longrightarrow} S_{1} \stackrel{p_{2}}{\longrightarrow} S_{2} \cdots \stackrel{p_{k}}{\longrightarrow} S_{k}$ in the state diagram corresponds to a latency sequence $\left\{p_{1}, p_{2}, \cdots, p_{k}\right\}$. The latency sequence represents $k+1$ initiations that are made at time steps $0, p_{1},\left(p_{1}+p_{2}\right), \cdots,\left(p_{1}+p_{2}+\cdots+p_{k}\right)$. In modulo scheduling, these time steps correspond to the offset values

$$
0, p_{1},\left(p_{1}+p_{2}\right) \bmod \mathbf{I I}, \cdots,\left(p_{1}+p_{2}+\cdots+p_{k}\right) \bmod \mathbf{I I}
$$

in a repetitive kernel. For example, the path $S_{1} \stackrel{2}{\longrightarrow} S_{2} \stackrel{2}{\longrightarrow} S_{3} \stackrel{2}{\longrightarrow} S_{4}$ corresponds to initiations at time steps $(0,2,4,6)$. These offset values for the path 
are collectively referred to as offset set, or in short form OffSet. Once the state diagram is constructed, then the OffSets corresponding to various paths can be used to guide the enhanced Co-Scheduling method to make quick and "good" decision about when (at what offset value) to schedule an instruction in the pipeline [7].

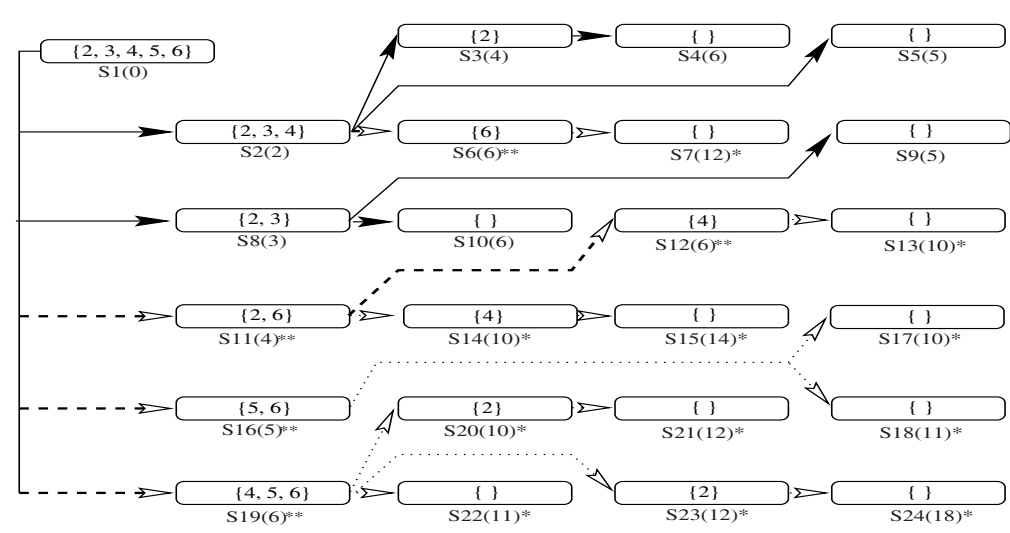

Fig. 2. State Diagram for the Example Reservation Table

\subsection{Problem Formulation}

The major challenge in the construction of a state diagram is that it can become extremely huge, consisting several million states, even for moderate values of II. For example, for the DEC Alpha architecture, the state diagram consists of 10,648 , and 224,400 paths when II is varied from 1 to 3 ! Fortunately, several paths in the state diagram are shown to be redundant $[7,8]$ in the sense that the corresponding $\mathrm{OffSets}$ are equal. In the above 3 state diagrams for DEC Alpha, only 3,9 , and 30 paths are distinct.

For the state diagram shown in Figure 2, the paths $S_{1} \stackrel{2}{\longrightarrow} S_{2} \stackrel{2}{\longrightarrow} S_{3} \stackrel{2}{\longrightarrow} S_{4}$ and the path $S_{1} \stackrel{4}{\longrightarrow} S_{11} \stackrel{6}{\longrightarrow} S_{14} \stackrel{4}{\longrightarrow} S_{15}$ correspond to the same OffSet $(0,2,4,6)$, and hence one of them is redundant. The reduced state diagram construction (RD) method proposed in [7] recognizes and eliminates redundant paths by making a simple observation. All states which correspond to initiation times $^{2}$ greater than II are pruned. Consider the the state $S_{14}$ which corresponds to an initiation at time 10 (shown in brackets adjacent to the state number in Figure 2). In Figure 2, these states (e.g., $S_{7}, S_{13}$, and $S_{14}$ ) are marked with a '*' and the arcs leading to these states are shown as dotted lines. Those marked with a '**' (the arcs leading to them are dashed lines) are also redundant states.

\footnotetext{
${ }^{2}$ Initiation time is the sum of the latency values from $S_{0}$ to that state along the path. Initiation time modulo II corresponds to an offset value.
} 
The RD method is unable to recognize them. This makes it somewhat inefficient in removing the redundancy. What's worse for the RD method is that it cannot completely eliminate all redundant paths. This is especially so for architectures involving multiple instruction types where different function units may share some of the resources, a common feature in real architectures.

In order for state diagram based approach to be useful in real compilers, it is important to deal with the redundancy-elimination in an efficient way. Further, even after eliminating all redundant paths, the state diagram may still consist of a large number of distinct paths. Hence, for practical reasons, the most important is to construct a large subset of distinct OffSets within a short time.

In this paper we propose two efficient solutions, the E-MIS method and the $\mathrm{RP}$ method, both of which are proved to be successful in attacking the above problem.

\section{A Graph Theoretic Approach for OffSets Generation}

In this section we relate the generation of OffSets to a well-know graph theory problem which facilitates the use of an existing algorithm as a direct and efficient method. This is based on a correspondence between the set of OffSets and the maximal compatibility classes which was established in [7].

First, we denote the set of permissible offset values by $\mathcal{O}=\left\{o_{0}, o_{1}, \cdots, o_{n-1}\right\}$ which includes all initial permissible latencies and 0 [7]. For the example discussed in Section 2.1, the set of permissible offset values is $\mathcal{O}=\{0,2,3,4,5,6\}$. The following definitions are useful in the discussion.

Definition 1. A path $S_{0} \stackrel{p_{1}}{\longrightarrow} S_{1} \stackrel{p_{2}}{\longrightarrow} S_{2} \cdots \stackrel{p_{k}}{\longrightarrow} S_{f}$ in the state diagram is called primary if the sum of the latency values does not exceed II, i.e., $p_{1}+p_{2}+\cdots+$ $p_{k}<$ II. A path is called secondary if $p_{1}+p_{2}+\cdots+p_{k}>$ II.

Definition 2. A compatibility class $\mathcal{C}$ (with respect to $\mathcal{O}$ ) is a subset of $\mathcal{O}$ such that for any pair of elements $c_{1}$ and $c_{2}$ in $\mathcal{C},\left(c_{1}-c_{2}\right) \bmod$ II is in $\mathcal{O}$. A compatibility class is maximal if it is not a proper subset of any other compatibility class.

Two compatible classes for $\mathcal{O}$ are $\{0,2,4,6\}$ and $\{0,2,5\}$. These compatibility classes are maximal.

Lemma 1. The OffSet of any path from the start state $S_{0}$ to the final state $S_{f}$ in the state diagram forms a maximal compatibility class of $\mathcal{O}$.

Lemma 2. For each maximal compatibility class $C$ of permissible offset values, there exists a primary path in the state diagrams whose OffSet $O$ is equal to $C$.

Hence obtaining all maximal compatible classes is a direct way of obtaining the OffSets. In order to obtain all maximal compatible classes of $\mathcal{O}$, we represent the compatibility information (between pairs of permissible offset values) in the 
form of an interference graph. The interference graph $G$ for $\mathcal{O}$ has $n$ vertices, each vertex representing one permissible offset value in $\mathcal{O}$. Two vertices $v_{1}$ and $v_{2}$ in $G$ are connected by an edge, if the corresponding offset values are not compatible. It is possible that a vertex may not share an edge with any other vertex in the graph. The offset value corresponding to such a vertex is compatible with other permissible offset values.

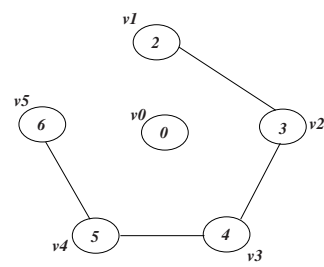

Fig. 3. Interference Graph for Permissible Offset Set $\{0,2,3,4,5,6\}$

The interference graph for the permissible offset values of our example, $\mathcal{O}=$ $\{0,2,3,4,5,6\}$ is shown in Figure 3. The offset value corresponding to each vertex is shown in circle in the figure. There is an edge between vertex $v_{1}$ (corresponding to offset 2) and $v_{2}$ (corresponding to offset 3 ) as 2 and 3 are not compatible in $\mathcal{O}$. Next we state the definitions of maximal independent sets from [5].

Definition 3. A subset $S$ of vertices is an independent set, if there exists no edge between any two vertices in $S$. An independent set is said to be maximal, if it is not contained in any other independent set.

In our example graph, $\left\{v_{0}, v_{1}, v_{3}, v_{5}\right\}$ and $\left\{v_{0}, v_{2}, v_{4}\right\}$ are maximal independent sets.

From the above definitions and the description of the interference graph, it clearly follows that each maximal independent set in the graph corresponds to a maximal compatibility class, and hence an OffSet in the reduced state diagram. Thus to generate the set of all OffSets of the state diagram, one needs to enumerate all maximal independent sets in the interference graph.

An efficient algorithm for this is reported in [18]. This enumeration of MIS is a direct method for the generation of all OffSets. An attractive features of this approach is that it does not incur any redundancy in the generation of OffSets. The complexity of the E-MIS method is $O(\alpha \cdot \beta \cdot \gamma)$ where $\alpha, \beta$ and $\gamma$ represent, respectively, the number of vertices, the number of edges, and the number of MIS present in the given graph. The space complexity of the algorithm is $O(\alpha+\beta)$. The number of vertices $\alpha$ in the interference graph is equal to $n$, the number permissible offset values. Further, since each edge in the graph represents non-compatibility between a pair of offset values, there can be at most $O\left(n^{2}\right)$ edges. Lastly an upper bound for $\gamma$ can be obtained by further relating 
maximal compatibility classes to the largest antichain ${ }^{3}$ of powerset of $\mathcal{O}$ [11]. The cardinality of the largest antichain is given by $\left(\begin{array}{c}n \\ \lfloor n / 2\rfloor\end{array}\right)$. Not surprisingly, this bound on $\gamma$ is exponential in $n$.

\section{Redundancy Prevention Method for State Diagram Construction}

In this section, we present the details of the RP method which exploits the the structure of the state diagram construction to identify and eliminate redundancy at the earliest opportunity. Further an attractive feature of the RP method is that it follows an aggressive approach for redundance elimination, even though this may mean missing a few OffSets. However our experimental results show that this aggressiveness pays well in state diagrams for real architecture without much loss of information.

\subsection{The Redundancy Prevention Algorithm}

In this discussion we will assume that the construction of the state diagram proceeds top down, in a depth-first fashion. In our example state diagram (Figure 2), consider the subtrees with their roots at $S_{6}, S_{11}, S_{16}$, and $S_{19}$. All paths in these subtrees are redundant. Thus if our construction method can somehow identify these states and eliminate them before the subtrees rooted on these state are constructed, then the redundant paths through these states can be eliminated.

If we carefully study the example state diagram, we find an interesting characteristic for these four states - the issue time or offset value of a state $S$ appears in the subtree rooted at one of its siblings $S^{\prime}$. In particular, the sib$\operatorname{ling} S^{\prime}$ is constructed earlier than $S$ in the depth-first construction method. We refer to this sibling $S^{\prime}$ as a left sibling of state $S$. Notice that there could be more than one left sibling for state $S$. For convenience, we use the term "left sibling subtree" for the tree rooted at a left sibling. For instance, subtrees rooted at $S_{2}$ and $S_{8}$ are the left sibling subtrees of state $S_{11}$.

If we inspect this property - the offset value of a state $S$ appearing in the left-sibling subtree - a little more carefully it reveals the fact that the partial OffSet, consisting of offset values for all states from the start state to $S$, is a subset of the OffSet for another path in the left-sibling subtree. Note that all paths through state $S$ will have this same partial offSet. Further, as the values in an OffSet are mutually compatible, the partial OffSet determines, to a great extent, what other values could be in the OffSets for the paths through $S$. As a consequence, it is very likely that all these paths could be redundant, and hence can be eliminated by not generating the state $S$ and the subtree rooted at $S$. The RP method aggressively removes these states to prevent redundancy. Note, however, it is only likely that all paths through $S$ are redundant. In other words,

\footnotetext{
${ }^{3}$ An antichain $A$ is a subset of $S$ such that for any two elements $a_{1}$ and $a_{2}$ in $A, a_{1}$ is not a proper subset of $a_{2}$.
} 
a state that satisfies the above property may have a path that is not redundant. We defer a discussion on this to Section 4.2. Based on the above observation, we introduce the following constraint that is used in the RP method.

Definition 4 (Redundancy Constraint). A state $S$ is said to satisfy the redundancy constraint $(R C)$ if the issue time of state $S$ is the same as that of a state $S^{\prime}$ and $S^{\prime}$ has occurred in one of the left sibling subtrees of $S$.

The basic steps in the RP method are:

(1) Follow the depth-first construction rule to construct the state diagram.

(2) If a given state $S$ has already created $k$ child states $S_{1}, S_{2}, \cdots, S_{k}$ and is going to create a new child state $S_{k+1}$ (it should be noted here that, at this time, all states in the subtrees rooted at $S_{1}, S_{2}, \cdots, S_{k}$ have been constructed), the redundancy constraint checks whether the issue time (offset value) of $S_{k+1}$ has occurred in subtrees rooted at $S_{1}, S_{2}, \cdots, S_{k}$. If so, the state $S_{k+1}$ will not be created; else it is added to the state diagram.

Step 2 sounds time-consuming because for each new child state to be created we need to check all states in the left sibling subtrees. It should be noticed here that the range of offset values is fixed, i.e., from 0 to II - 1. Therefore, it is not difficult to construct an implementation for the RP method the RC check could be done as a simple table lookup. The table, called "left_sibling_states" records the issue time of all states in the left sibling subtrees. The size of this table depends on the model, but is very small. Further, for computational efficiency, the RP method constructs the state diagram as a tree rather than as a directed acyclic graph [7]. This means that certain states, e.g., the final state of the state diagram which contains an empty permissible latency set may be repeated several times (states $S_{5}, S_{9}$, and $S_{10}$ ) as shown in Figure 2.

The attractive features of this approach are that it exploits the structure of state diagram construction (top-down and left-to-right) to eliminate redundancy at the earliest opportunity. Second, the RP method is aggressive in redundance elimination. Lastly, as a comparison, the E-MIS method is an exact method, and hence has the overhead of having to construct the complete interference graph, before the generation of 0 ffSets.

\subsection{Properties of RP Method}

Note that the RP method uses the redundancy constraint to eliminate states that could possibly lead to redundant paths. This raises two questions: (i) Does the RP method eliminate all redundant paths? (ii) Will the RP method ever eliminate a path that is non-redundant? We answer these two questions in this subsection.

Theorem 1. There are no redundant paths in the state diagram tree constructed by the RP method. 
The proof of this theorem proceeds by showing that, at any point in time, the partial state diagram tree constructed so far does not have any redundancy. Due to the limitation on paper length, we could not give the proof here in this paper.

We remark that the RP method uses the redundancy constraint which is only a necessary, but not sufficient condition. As a result it may miss some non-redundant paths due to the aggressive pruning employed by this method. However, we argue that there is still a good chance that of the "missed" OffSets will reappear in some other paths of the state diagram. In Section 5 we study empirically how many non-redundant paths does RP miss in the state diagram and whether they have any influence on the constructed software pipelined schedule.

\section{$5 \quad$ Experimental Results}

In this section we report our experience in generating the OffSets using the proposed methods for various values of II for two real processors, namely the DEC Alpha 21064 superscalar processor and the Cydra VLIW processor. Both architectures have complex resource usage pattern with sharing of resources. The $\mathrm{RP}$ and the E-MIS methods have been implemented in our Modulo Scheduling Testbed (MOST) framework [1].

\subsection{Construction Time Comparisons}

In order to compare the construction speed of RP, E-MIS and RD methods in a fair manner, all the three methods were run to generate a large subset of OffSets, consisting of the first, say, 100,000 distinct OffSets. Tables 3 compares the construction time for OffSets generation for the three methods on an Ultra-450 Workstation (with a $250 \mathrm{MHz}$ clock). We observe that the RD method is much slower than RP and E-MIS. For example, the RD method failed to generate 100,000 distinct OffSets even after running for 24 hours for the Alpha architecture, for an $\mathbf{I I}=8$. Hence for larger values of II, we only compared the $\mathrm{RP}$ and the E-MIS methods.

Figure 4 shows the normalized (w.r.t. the RP method) construction time taken by the E-MIS methods for various values of II for the two architectures. We observe that the RP and the E-MIS methods are competitive: E-MIS performs better for small values of II while RP performs better for moderate to large IIs. This is not surprising as RP is a very efficient heuristics that we employ to get the OffSets quickly while the E-MIS method, being inherently an exact method, is slow, especially for large values II. This is due to the fact that the E-MIS method needs to construct the entire (large) interference graph before the generation of OffSets.

\subsection{How many Paths Does RP Miss?}

As mentioned earlier the RP method can miss some non-redundant paths during the construction. It is important to verify that RP will not miss too much 

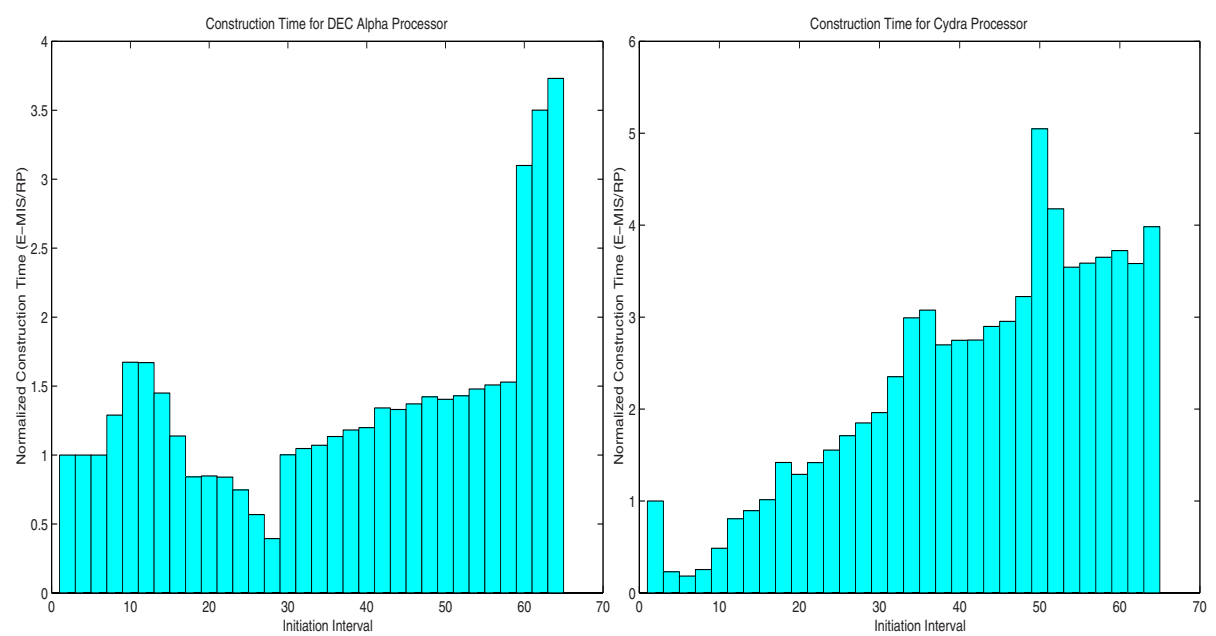

Fig. 4. Comparison of Construction Time for RP and E-MIS Methods

useful information. On the other hand, since E-MIS is capable of generating all distinct $\mathrm{OffSets}$, we can compare the number of OffSets generated by these two methods if these methods were allowed to complete their execution without any restriction on the maximum number of 0 ffSets generated. The number of distinct OffSets in the state diagram is significantly large, even for small values of II. Further the construction time grows exponentially with larger values of II. This has limited our experiments to values of II less than 16 for the Alpha processor and less than 12 for the Cydra processor. The results are tabulated in Table 1. From the experimental results, we observe that the RP method generates all non-redundant paths in the considered cases. It didn't miss a single path! This is somewhat surprising to us, as we anticipated that the RP heuristic to eliminate some non-redundant paths as well. However, we believe that the RP method may still miss some non-redundant paths for large values of II and/or for other architectures.

\subsection{Application to Co-scheduling}

Lastly we attempt to answer the question how critical are the missed OffSets, if any, in terms of the quality of the constructed software pipelined schedule, when the the enhanced Co-Scheduling method uses the OffSets generated by the RP method. Since the RP method did not miss any path for the DEC Alpha and Cydra processor (for the values of II that we could experiment), its application in the enhanced Co-Scheduling should perform at least as good as any other OffSet generation method, e.g., E-MIS or RD methods, applied to Co-Scheduling. What happens if the RP method does miss some OffSets? To answer to this question, we considered the reservation tables used in [7] which 


\begin{tabular}{|r|r|r|r|r|r|r|}
\hline II & \multicolumn{3}{|c|}{ DEC Alpha Processor } & \multicolumn{3}{|c|}{ Cydra Processor } \\
\cline { 2 - 6 } & \multicolumn{2}{|c|}{$\begin{array}{r}\text { No. of Paths } \\
\text { Generated }\end{array}$} & $\begin{array}{r}\text { No. of } \\
\text { Missed }\end{array}$ & \multicolumn{2}{|c|}{$\begin{array}{c}\text { No. of Paths } \\
\text { Generated }\end{array}$} & $\begin{array}{r}\text { No. of } \\
\text { Missed }\end{array}$ \\
\cline { 2 - 6 } & RP & E-MIS & Paths & RP & E-MIS & Paths \\
\hline 1 & 3 & 3 & 0 & 4 & 4 & 0 \\
2 & 9 & 9 & 0 & 16 & 16 & 0 \\
3 & 30 & 30 & 0 & 64 & 64 & 0 \\
4 & 93 & 93 & 0 & 256 & 256 & 0 \\
5 & 288 & 288 & 0 & 1,024 & 1,024 & 0 \\
6 & 894 & 894 & 0 & 4,096 & 4,096 & 0 \\
7 & 2,775 & 2,775 & 0 & 131,072 & 131,072 & 0 \\
8 & 8,613 & 8,613 & 0 & 589,824 & 589,824 & 0 \\
9 & 26,733 & 26,733 & 0 & $7,340,032$ & $7,340,032$ & 0 \\
10 & 82,974 & 82,974 & 0 & $32,505,856$ & $32,505,856$ & 0 \\
11 & 257,535 & 257,535 & 0 & $142,606,336$ & $142,606,336$ & 0 \\
12 & 799,338 & 799,338 & 0 & $\mathrm{~N} / \mathrm{A}$ & $\mathrm{N} / \mathrm{A}$ & $\mathrm{N} / \mathrm{A}$ \\
13 & $2,480,988$ & $2,480,988$ & 0 & $\mathrm{~N} / \mathrm{A}$ & $\mathrm{N} / \mathrm{A}$ & $\mathrm{N} / \mathrm{A}$ \\
14 & $7,700,499$ & $7,700,499$ & 0 & $\mathrm{~N} / \mathrm{A}$ & $\mathrm{N} / \mathrm{A}$ & $\mathrm{N} / \mathrm{A}$ \\
15 & $23,900,835$ & $23,900,835$ & 0 & $\mathrm{~N} / \mathrm{A}$ & $\mathrm{N} / \mathrm{A}$ & $\mathrm{N} / \mathrm{A}$ \\
16 & $74,183,493$ & $74,183,493$ & 0 & $\mathrm{~N} / \mathrm{A}$ & $\mathrm{N} / \mathrm{A}$ & $\mathrm{N} / \mathrm{A}$ \\
\hline
\end{tabular}

Table 1. Missed Paths in the RP Method

model function units with complex resource usage, but without any sharing of resources. In these reservation tables the RP method misses a few paths even for small values of II. For example, for one of the reservation tables, the RP method generated only 22 out of 26 distinct OffSets. Also, to reduce the complexity of the enhanced Co-Scheduling method, the scheduling method used all distinct OffSets, up to a maximum of 1000, generated either by RP or E-MIS method. The enhanced Co-Scheduling was applied to a set of 737 loops consisting of single basic block extracted from scientific benchmark programs. We compare the constructed software pipelined schedules, in terms of the initiation interval and the construction time for schedule in Table 2. In order to have a fair comparison, we did not include the time for the generation of OffSets in the scheduling time. We observe that both methods perform more or less alike. This once again establishes that the OffSets missed by the RP method are not really critical in terms of the quality of the constructed schedule.

\subsection{Summary of Results}

To summarize our experimental results:

- The RD method is too slow in the construction of state diagram for architectures in which function units share resources. Hence it is impractical to apply this for modeling real processors. 


\begin{tabular}{|c|c|c|c|c|c|}
\hline Measure & $\begin{array}{r}\text { RP-Based Co-Scheduling } \\
\text { Method Better }\end{array}$ & $\begin{array}{r}\text { E-MIS-Based Co-Scheduling } \\
\text { Method Better }\end{array}$ & $\begin{array}{c}\text { Both } \\
\text { Same }\end{array}$ \\
\cline { 2 - 6 } & \# Cases \% Improvement & \# Cases & \% Improvement & \# Cases \\
\hline Initiation Interval & 18 & $1.11 \%$ & 16 & $0.16 \%$ & 703 \\
\hline Scheduling Time & 289 & $94.18 \%$ & 448 & $94.4 \%$ & 0 \\
\hline
\end{tabular}

Table 2. Effectiveness of RP and E-MIS in Software Pipelining

- Both the RP and E-MIS methods are much faster than RD, by 3 to 4 orders of magnitude, and their construction time is acceptable for implementation in real compilers.

- In terms of construction speed, the E-MIS method performs better for small values of II while RP method performs better for large II values.

- Considering the overall performance, for a real compiler, it is better to choose RP method because RP behaves better for large II values. A 3-fold speedup in large II values could mean a reduction of tens of minutes of construction time. For small II values, however, a 3-fold speedup may only mean a reduction of several seconds in construction time.

- Though the RP method can not guarantee to always generate all distinct OffSets, we found that it is capable of generating all non-redundant $0 f f$ Sets in the experiments that we conducted. Further, we found that the generated OffSets when applied to the Co-Scheduling method can get equally good performance, in terms of the constructed schedule.

\section{Conclusions}

In this paper we have proposed two efficient methods to construct distinct paths in the state diagram used for modeling complex pipeline resource usage in software pipelining. The methods proposed in this paper, namely the E-MIS method and the RP method completely eliminate the generation of redundant paths. The first of these methods, the E-MIS method is obtained by relating the OffSets generation to a well-known graph theoretic problem, viz., the enumeration of maximal independent sets of an interference graph. The second method, the RP method, uses a simple but very effective heuristic to prevent the construction of states that may cause redundant paths. We formally establish that this heuristic results in redundance-free state diagram. We compare the performance of RP and E-MIS methods in modeling two real processors, namely the DEC Alpha processor and the Cydra VLIW processor. The RP and E-MIS methods were found to be much superior than the RD method, by 3 to 4 orders of magnitude. When compared between themselves, the RP and the MIS methods perform competitively, the RP performing better for larger values of II while the E-MIS performing better for small II. Lastly, we have applied the OffSets generated by these methods in the enhanced Co-Scheduling method and reported their performance. 


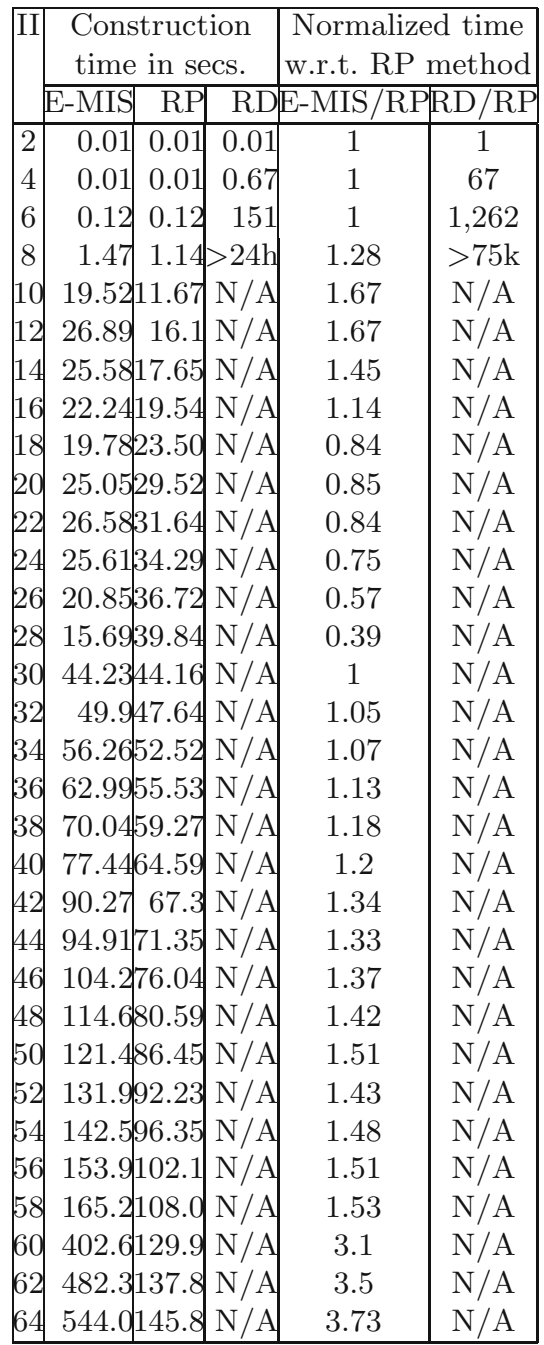

DEC Alpha Processor

\begin{tabular}{|c|c|c|c|c|c|}
\hline \multirow[t]{2}{*}{11} & \multicolumn{3}{|c|}{$\begin{array}{l}\text { Construction } \\
\text { time in secs. }\end{array}$} & \multicolumn{2}{|c|}{\begin{tabular}{|c|} 
Normalized time \\
w.r.t. RP method
\end{tabular}} \\
\hline & E-MIS & $\mathrm{RP}$ & $\mathrm{RD}$ & E-MIS/RF & $\mathrm{RD} / \mathrm{RP}$ \\
\hline 2 & 0.01 & 0.01 & 4296 & 1 & 429,700 \\
\hline 4 & 0.03 & 0.13 & $>24 \mathrm{~h}$ & 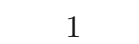 & $664 \mathrm{k}$ \\
\hline 6 & 0.39 & 2.13 & $\mathrm{~N} / \mathrm{A}$ & 0.17 & $\mathrm{~N} / \mathrm{A}$ \\
\hline 8 & 15.37 & 60.4 & $\mathrm{~N} / \mathrm{A}$ & 0.25 & $\mathrm{~N} / \mathrm{A}$ \\
\hline 10 & 41.03 & 84.4 & $\mathrm{~N} / \mathrm{A}$ & 0.48 & $\mathrm{~N} / \mathrm{A}$ \\
\hline 12 & 77.2 & 95.7 & $\mathrm{~N} / \mathrm{A}$ & 0.81 & $\mathrm{~N} / \mathrm{A}$ \\
\hline 4 & 95.991 & 107.4 & $\mathrm{~N} / \mathrm{A}$ & 0.89 & $\mathrm{~N} / \mathrm{A}$ \\
\hline 16 & 121.2 & 9.4 & $\mathrm{~N} / \mathrm{A}$ & 1.01 & $\mathrm{~N} / \mathrm{A}$ \\
\hline 18 & 173.01 & 122.0 & $\mathrm{~N} / \mathrm{A}$ & 1.41 & $\mathrm{~N} / \mathrm{A}$ \\
\hline 20 & 180.61 & 140.1 & $\mathrm{~N} / \mathrm{A}$ & 1.29 & $\mathrm{~N} / \mathrm{A}$ \\
\hline 22 & 219.41 & 154.8 & $\mathrm{~N} / \mathrm{A}$ & 1.42 & $\mathrm{~N} / \mathrm{A}$ \\
\hline 24 & 259.01 & 166.7 & $\mathrm{~N} / \mathrm{A}$ & 1.55 & $\mathrm{~N} / \mathrm{A}$ \\
\hline 26 & 302.11 & 176.7 & $\mathrm{~N} / \mathrm{A}$ & 1.71 & $\mathrm{~N} / \mathrm{A}$ \\
\hline 28 & 350.31 & 189.5 & $\mathrm{~N} / \mathrm{A}$ & 1.84 & $\mathrm{~N} / \mathrm{A}$ \\
\hline 30 & 399. & 203.6 & $\mathrm{~N} / \mathrm{A}$ & 1.96 & $\mathrm{~N} / \mathrm{A}$ \\
\hline 32 & 511.12 & 217.3 & $\mathrm{~N} / \mathrm{A}$ & 2.35 & $\mathrm{~N} / \mathrm{A}$ \\
\hline 34 & 666.32 & 222.7 & $\mathrm{~N} / \mathrm{A}$ & 2.99 & $\mathrm{~N} / \mathrm{A}$ \\
\hline 36 & 759.62 & 247.0 & $\mathrm{~N} / \mathrm{A}$ & 3.08 & $\mathrm{~N} / \mathrm{A}$ \\
\hline 38 & 713.52 & 264.4 & $\mathrm{~N} / \mathrm{A}$ & 2.7 & $\mathrm{~N} / \mathrm{A}$ \\
\hline 40 & 767.32 & 279.3 & $\mathrm{~N} / \mathrm{A}$ & 2.74 & $\mathrm{~N} / \mathrm{A}$ \\
\hline 42 & 810.42 & 294.7 & $\mathrm{~N} / \mathrm{A}$ & 2.75 & $\mathrm{~N} / \mathrm{A}$ \\
\hline 44 & 893.63 & 308.3 & $\mathrm{~N} / \mathrm{A}$ & 2.9 & $\mathrm{~N} / \mathrm{A}$ \\
\hline 46 & 962.6 & 325.9 & $\mathrm{~N} / \mathrm{A}$ & 2.95 & $\mathrm{~N} / \mathrm{A}$ \\
\hline 48 & 1106.23 & 343.1 & $\mathrm{~N} / \mathrm{A}$ & 3.22 & $\mathrm{~N} / \mathrm{A}$ \\
\hline & 1735.53 & 343.8 & $\mathrm{~N} / \mathrm{A}$ & 5.04 & $\mathrm{~N} / \mathrm{A}$ \\
\hline & 1579.53 & 378.1 & $\mathrm{~N} / \mathrm{A}$ & 4.18 & $\mathrm{~N} / \mathrm{A}$ \\
\hline & 1392.03 & 392.9 & $\mathrm{~N} / \mathrm{A}$ & 3.54 & $\mathrm{~N} / \mathrm{A}$ \\
\hline & 1478.94 & 412.2 & $\mathrm{~N} / \mathrm{A}$ & 3.59 & $\mathrm{~N} / \mathrm{A}$ \\
\hline & 1573.44 & 131.0 & $\mathrm{~N} / \mathrm{A}$ & 3.65 & $\mathrm{~N} / \mathrm{A}$ \\
\hline & 1675.54 & 150.0 & $\mathrm{~N} / \mathrm{A}$ & 3.72 & $\mathrm{~N} / \mathrm{A}$ \\
\hline & 1773.54 & 195.1 & $\mathrm{N} / \mathrm{A}$ & 3.58 & $\mathrm{~N} / \mathrm{A}$ \\
\hline & 1970.54 & 494.8 & $\mathrm{~N} / \mathrm{A}$ & 3.98 & $\mathrm{~N} / \mathrm{A}$ \\
\hline
\end{tabular}

Cydra Processor

Table 3. Comparison of Construction Time for the 3 Methods

\section{References}

1. E. R. Altman. Optimal Software Pipelining with Function Unit and Register Constraints. Ph.D. thesis, McGill U., Montréal, Qué., Oct. 1995.162

2. V. Bala and N. Rubin. Efficient instruction scheduling using finite state automata. In Proc. of the 28th Ann. Intl. Symp. on Microarchitecture, pages 46-56, Ann Arbor, MI, Nov. 29-Dec.1, 1995. 154, 155 
3. G. Beck, D.W.L. Yen, and T. L. Anderson. The Cydra-5 minisupercomputer: Architecture and implementation. Journal of Supercomputing, 7, May 1993. 153, 155

4. A. E. Eichenberger and E. S. Davidson. A reduced multipipeline machine description that preserves scheduling constraints. In Proc. of the ACM SIGPLAN '96 Conf. on Programming Language Design and Implementation, pages 12-22, Philadelphia, PA, May 21-24, 1996. 155

5. M.C. Golumbic. Algorithmic Graph Theory and Perfect Graphs. Academic Press, New York, 1980. 159

6. R. Govindarajan, Erik R. Altman, and Guang R. Gao. Co-scheduling hardware and software pipelines. In Proc. of the Second Intl. Symp. on High-Performance Computer Architecture, pages 52-61, San Jose, CA, Feb. 3-7, 1996. IEEE Computer Society. 154, 155, 156

7. R. Govindarajan, N.S.S. Narasimha Rao, E. R. Altman, and G. R. Gao. An enhanced co-scheduling method using reduced ms-state diagrams. In Proc. of the 12th Intl. Parallel Processing Symp., Orlando, FL, Mar. 1998. IEEE Computer Society. Merged with 9th Intl. Symp. on Parallel and Distributed Processing. 154, $156,157,158,161,163$

8. R. Govindarajan, N.S.S. Narasimha Rao, Erik R. Altman, and Guang R. Gao. An enhanced co-scheduling method using reduced ms-state diagrams. CAPSL Technical Memo 17, Dept. of Electrical \& Computer Engineering, University of Delaware, Newark 19716, U.S.A., Feb. 1998. Also as Tech. Report TR-98-06, Dept. of Computer Science \& Automation, Indian Institute of Science, Bangaloe, 560 012, India. (available via http://www.csa.iisc.ernet.in/ govind/papers/TR-98-2.ps.gz). 154, 156, 157

9. R. A. Huff. Lifetime-sensitive modulo scheduling. In Proc. of the ACM SIGPLAN '93 Conf. on Programming Language Design and Implementation, pages 258-267, Albuquerque, New Mexico, June 23-25, 1993. 153, 155, 156

10. M. Lam. Software pipelining: An effective scheduling technique for VLIW machines. In Proc. of the SIGPLAN '88 Conf. on Programming Language Design and Implementation, pages 318-328, Atlanta, Georgia, June 22-24, $1988 . \quad 153,155$, 156

11. C.L. Liu. Introduction to Combinatorial Mathematics. McGraw-Hill Book Co., New York, NY, 1968. 160

12. Thomas Muller. Employing finite state automata for resource scheduling. In Proc. of the 26th Ann. Intl. Symp. on Microarchitecture, Austin, TX, Dec. 1-3, 1993. 154

13. J. H. Patel and E. S. Davidson. Improving the throughput of a pipeline by insertion of delays. In Proc. of the 3rd Ann. Symp. on Computer Architecture, pages 159-164, Clearwater, FL, Jan. 19-21, 1976. 156

14. T. A. Proebsting and C. W. Fraser. Detecting pipeline structural hazards quickly. In Conf. Record of the 21st ACM SIGPLAN-SIGACT Symp. on Principles of Programming Languages, pages 280-286, Portland, OR, Jan. 17-21, 1994. 154, 155

15. B. R. Rau and J. A. Fisher. Instruction-level parallel processing: History, overview and perspective. Journal of Supercomputing, 7:9-50, May 1993. 153, 155, 156

16. B. R. Rau. Iterative modulo scheduling: An algorithm for software pipelining loops. In Proc. of the 27th Ann. Intl. Symp. on Microarchitecture, pages 63-74, San Jose, California, November 30-December2, 1994. 153, 155, 156 
17. John Ruttenberg, G. R. Gao, A. Stouchinin, and W. Lichtenstein. Software pipelining showdown: Optimal vs. heuristic methods in a production compiler. In Proc. of the ACM SIGPLAN '96 Conf. on Programming Language Design and Implementation, pages 1-11, Philadelphia, PA, May 21-24, 1996. 153

18. S. Tsukiyama, M. Ide, H. Ariyoshi, and I. Shirakawa. A new algorithm for generating all the maximal independent sets. SIAM Jl. on Computing, 6(3):505-517, Sep. 1977. 159 